\title{
Effects of Fracture Parameters in an Anisotropy Model on P-Wave Azimuthal Amplitude Responses
}

\author{
Fatkhan \\ Program Studi Teknik Geofisika FIKTM-ITB
}

\begin{abstract}
The study showed that the presence of vertically aligned fractures in reservoir rock could possibly be inferred through analysis of P-wave reflectivity variations with azimuth. The azimuthal amplitude responses measured at an interface separating overburden anisotropy and fractured rock rely on several parameters that can be decomposed into the background rock and the fracture contributions.

The effect of fracture porosity on P-wave azimuthal amplitude response is small for moderate incidence angles. For fluid filled fractures P-wave anisotropy is mildly affected. For dry fractures the difference in P-wave anisotropy between low and high fracture porosities is high. This suggests that an assumption of overburden isotropy may result in a large error in predicting the fluid type and saturation.
\end{abstract}

Keywords: Azimuthal anisotropy; VTI; HTI; Linear Slip Method; Penny-shaped cracks.

\section{Introduction}

An anisotropic medium happens if a certain physical parameter changes with the direction of measurement. Anisotropic effects are usually observed at wide angles and/or multi-azimuthal seismic data in which a wave velocity changes with direction of propagation. Hence, there is concern to add important information that is being missed when assuming an isotropic Earth.

Numerical modelling techniques can provide information necessary for a better understanding of wave propagation in fractured media or more generally in azimuthal anisotropic media. Reflection amplitudes vary with angles of incidence, often referred to as amplitude versus offset (AVO), at an interface separating from isotropic or anisotropic media. These variations depend on the contrast in elastic properties between two layers.

Fractures often cause anisotropy in rocks in which are in general co-aligned with the dominant horizontal stress direction [3]. At depth, only sub-vertical or vertical fractures are likely to remain open and as such they affect seismic wave propagation. If a reservoir rock (typically sandstone) is pervaded by a system of

Received April 2006, Revised September $25^{\text {th }} 2006$, Accepted for publication September $25^{\text {th }} 2006$. 
vertical aligned fractures. Then it can be modeled as transverse isotropy with a horizontal axis of symmetry (HTI), or azimuthal anisotropy. The necessity for the inclusion of anisotropy into calculations of reflection amplitudes comes from the simple fact that shales, which are intrinsically anisotropic, are comprised mostly of sedimentary rocks and are also the most common sealing rocks. Reservoir rocks, in particular sandstones, are often fractured and possess a different symmetry class from shales.

Important information, associated with rock properties, is in general held in amplitude variations with offset or azimuth AVO or AVAz) but is often difficult to extract. Hence, reflection amplitudes could be analyzed by various methods such as by a generalized form of Zoeppritz's equations that is suitable for general anisotropy [8].This is achieved by employing the effective medium theory, such as linear-slip interface, penny-shaped crack.

The AVO response of a fractured reservoir may be further complicated by the anisotropy of the sealing rock. Shales, which are the most common sealing rocks, are intrinsically anisotropic with an axis of symmetry normal to the bedding (vertical transverse isotropy (VTI)). Hence shale (the VTI medium) overlying a vertically fractured reservoir sand (the HTI medium) is probably an important model for AVO investigations.

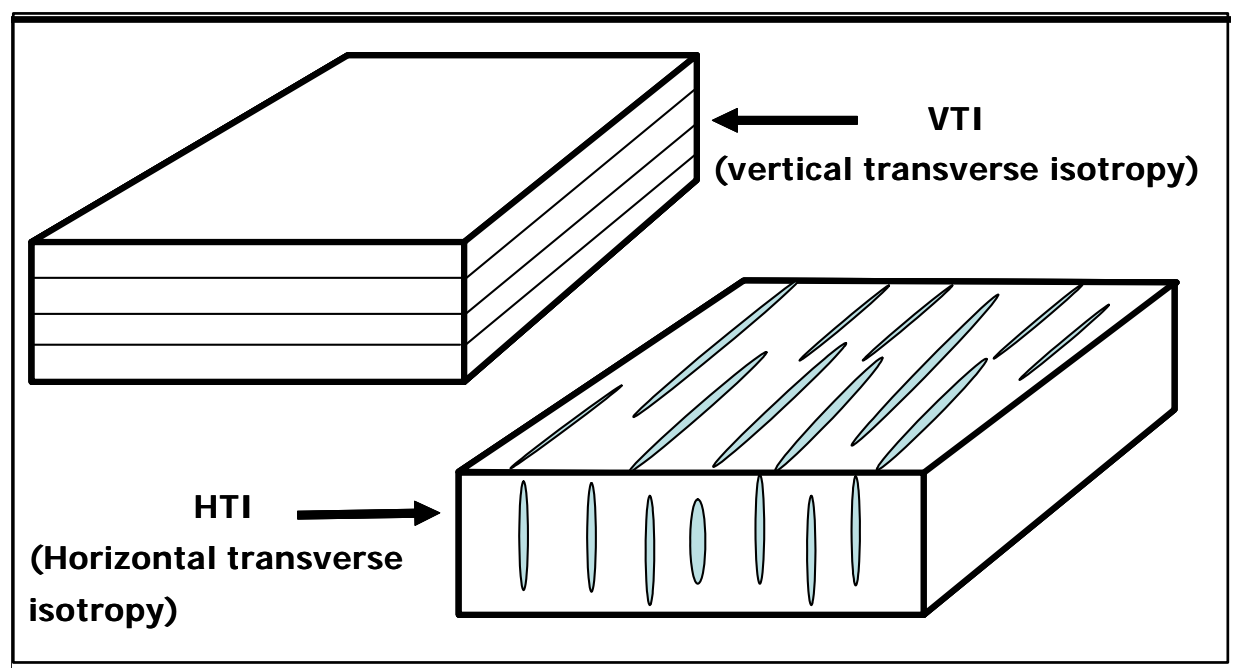

Figure 1 Anisotropy classes, the HTI and VTI media, are typically encountered at scale of seismic wavelengths. A commonly geologic model for the VTI medium is a massive shale layer; whilst for the HTI is a fracture reservoir. 
Backus [1] developed averaging techniques to determine the elastic properties of a heterogeneous solid having the same elastic properties as a homogeneous anisotropic elastic solid. This approach obviously has a great advantage since waves propagating in complicated geological models, such as ones with smallscale heterogeneities, can be replaced mathematically by simple geologic models with identical bulk properties. It is much easier to develop codes using simple models than complicated models.

Wave propagation in a medium containing aligned fractures may also be simulated under several assumptions of wave propagation in an elastic anisotropic solid [3]. The first step in this procedure is to determine effective elastic constants that have similar variations of properties as a fractured material. There are a number of theories for generating equivalent elastic constants for fractured media (e.g. [6], [4]).

Schoenberg [6] presented a method to generate the elastic tensor for a homogeneous medium containing fractures that he called the linear slip theory. In this approach, fractures are modelled as thin infinite parallel planes, highly compliant, non-rigid layers embedded in solid host rock and their shape and microstructure are not considered [2], [6]. The linear slip model is valid for the long wavelength limit. However, in the small wavelength limit reflections and transmissions can occur at every fracture. In this case wave propagation through a rock containing linear slip fractures can be dispersive.

In Hudson's theory [4], fractures are modelled as plane distributions of small isolated cracks, called penny-shaped crack model. The radii and the distances between adjacent fractures are assumed to be small compared to the wavelength, and the interaction between fractures is also assumed to be small. Hudson's theory predicts elastic anisotropy due to the presence of filled or empty ellipsoidal inclusions. Even though there are different assumptions to derive both theories of seismic wave propagation in fractured media, in some cases both theories can still be related.

\subsection{Linear Slip Model}

Schoenberg and Muir [7] developed a calculus that simplifies the combination of the effects of layering and fracturing. The calculus is shown to be applicable when a set of layers became infinitely thin and infinitely compliant, leading to a simple method of deriving the equivalent medium properties of an arbitrary anisotropic medium in which is embedded a set of parallel fractures. Each 
constituent in a finely layered medium and each set of fractures can be represented by separate elements in a transform domain. In that domain, elements can be combined using simple addition, assuming that there is no non linear interaction between constituent layers and/ or fracture sets.

According to Schoenberg and Muir [7], fractured rock may be modelled and decomposed into:

$$
\text { fractures }+ \text { rock } \longleftrightarrow \text { fractured rock }
$$

The effective compliance, $\mathrm{S}$, of fractured zone in the long wavelength limit is simply the sum of the background compliance $S_{b}$ and the excess fracture compliance $\mathrm{S}_{\mathrm{f}}$ [9]. The compliance $\mathrm{S}$ is by definition reciprocal to the stiffness C. The equation for the effective compliance of fractured rock can be described by the compliance matrix:

$$
\mathrm{S}=\mathrm{S}_{\mathrm{b}}+\mathrm{S}_{\mathrm{f}}
$$

where: $\quad \mathrm{S} \quad$ : the effective compliance of fractured rock.

$\mathrm{S}_{\mathrm{b}} \quad$ : the background compliance.

$\mathrm{S}_{\mathrm{f}} \quad$ : the excess fracture compliance.

\subsection{Penny-Shaped Crack Model}

Another alternative model for an effective medium theory of fractures is to be found in the concept of the penny shaped cracks [4]. The stiffness matrix $\mathrm{C}_{\mathrm{mn}}$ of the equivalent fractured medium for the first-order perturbations can be written as:

$$
\mathrm{C}_{\mathrm{mn}}=\mathrm{C}_{\mathrm{mn}}{ }^{0}+\mathrm{C}_{\mathrm{mn}}{ }^{1}+\mathrm{O}\left(\mathrm{e}^{2}\right)
$$

where

$\mathrm{C}_{\mathrm{mn}}{ }^{0}=$ the stiffness of the non-fractured matrix.

$\mathrm{C}_{\mathrm{mn}}{ }^{1}=$ The first order perturbations due to the fracture sets.

$\mathrm{O}\left(\mathrm{e}^{2}\right)=$ The higher order wave field scattering at the fractures.

\subsection{Relationships between Linear Slip and Penny-Shaped Crack Models}

Bakulin, et al. [2] showed that the penny-shaped crack model, under assumption of non-interacting fractures, is equivalent to the linear slip model of Schoenberg [6]. If the fractures are assumed to be invariant with respect to rotation about the axis normal to the fracture direction and their background is isotropic, the overall fracture compliance tensor depends on only two fracture compliance tensors, the fracture normal compliance tensor $\left(Z_{N}\right)$ and the tangential compliance tensor $\left(\mathrm{Z}_{\mathrm{T}}\right)$. These two fracture compliance tensors can be related to Hudson's fracture models. Schoenberg and Sayers [9] suggested that normal-to- 
tangential fracture compliance ratios $\left(\mathrm{Z}_{\mathrm{N}} / \mathrm{Z}_{\mathrm{T}}\right)$ may be used as an effective indicator of fluid type and possibly saturation. For dry fractures this ratio approaches unity, that is $Z_{N} / Z_{T} \approx 1$ for liquid-filled fractures, $Z_{N}$ is much smaller than $Z_{T}$, hence the ratio $Z_{N} / Z_{T}$ approaches zero. The relationships between the penny-shaped crack and the linear slip models are utilized, since the linear slip method does not provide explicit fracture parameters [2].

\section{$3 \quad$ Methods}

To see effects of changing fracture parameters on the azimuthal AVO responses of vertically fractured sands overlain by horizontally anisotropic shales, the following steps were required:

1. Set fracture density, aspect ratio and fluid properties via bulk and shear moduli.

2. Compute effective elastic constants using Hudson's penny-shape crack model.

3. Relate Hudson's penny shape crack model to Schoenberg's Linear Slip theory.

4. Compute effective elastic properties by adding the fracture compliance matrix to isotropic background compliances

5. Invert the compliance matrix to get the stiffness matrix and compute the azimuthal AVO response using anisotropic Zoeppritz solutions of Schoenberg and Protazio's.

6. Compute the solution for different azimuths by utilizing Bond transformations.

\section{$4 \quad$ Results}

\subsection{Azimuthal AVO Modelling for Anisotropic Shale/Fractured Sand Interface}

Following Rutherford and Williams, in isotropic AVO analysis reflection responses are often classified into three classes. Model parameters used for three classes were taken from Sayers and Rickett [5] and are given in Table 1. The following AVO modelling examples show the combined effects of an anisotropic shale/sand sequence across three sand classes. Introducing anisotropy in the overlying shale layer modifies the contrast of elastic parameters across the interface. Since the VTI medium is azimuthally invariant, the effect of shale on AVO responses only varies with incidence angles. 
Table 1 Model parameters used for classes 1, 2 and 3.

\begin{tabular}{lcccccc}
\hline & \multicolumn{2}{c}{ Class 1 } & \multicolumn{2}{c}{ Class 2 } & \multicolumn{2}{c}{ Class 3 } \\
\hline & Shale & Sand & Shale & Sand & Shale & Sand \\
\hline $\mathbf{V p}(\mathbf{k m} / \mathbf{s e c})$ & 3.30 & 4.20 & 2.96 & 3.49 & 2.73 & 2.02 \\
$\mathbf{V s}(\mathbf{k m} / \mathbf{s e c})$ & 1.70 & 2.70 & 1.38 & 2.29 & 1.24 & 1.23 \\
$\boldsymbol{\rho}(\mathbf{g r} / \mathbf{c c})$ & 2.35 & 2.49 & 2.43 & 2.14 & 2.35 & 2.13 \\
$\sigma($ Poisson ratio) & 0.32 & 0.15 & 0.36 & 0.12 & 0.37 & 0.2 \\
\hline
\end{tabular}

The VTI of shales was modelled with two different signs of Thomsen's anisotropic parameter $\delta$; negative and positive. In the first case, Thomsen's anisotropy parameters were $\delta=-0.15, \varepsilon=0.15$ and $\gamma=0.1$, while in the second case Thomsen's anisotropy parameters were $\delta=0.12, \varepsilon=0.15$ and $\gamma=0.1$. Fractures were modelled in terms of the linear slip theory by setting a tangential fracture weakness $\left(\delta_{\mathrm{T}}=0.2\right)$ and three different $Z_{\mathrm{N}} / \mathrm{Z}_{\mathrm{T}}$ ratios of 0.95 (dry fractures) and 0.05 (fluid-saturated).

P-wave AVO modelling for shale/sand system across all three classes is shown in Figure 2. A comparative display is used to illustrate the difference between isotropic shale/HTI sand and VTI shale/HTI sand responses. The anisotropy of the shale layer is modelled such that, in one case, parameter delta was positive $(\delta=0.12)$, while in the other case, this parameter had negative value $(\delta=-$ 0.15). Two directions are shown; orthogonal and along the fracture azimuth. Dry fractures are modelled. It is clear that the VTI overburden modified the AVO gradient in all cases since the anisotropic parameter $\delta$ has a strong effect on the near vertical P-wave velocity. In all cases and for both directions the anisotropy of shales considerably modified the AVO gradient. For negative $\delta$ the magnitude of the AVO gradient decreases compared to that of isotropic overburden. For positive $\delta$ the magnitude of the AVO gradient increases compared to that of isotropic overburden. This shows that anisotropy of shales can, if not accounted for, affect AVO interpretation considerably.

The P-wave AVO response for fluid saturated fractures overlain by isotropic and anisotropic shales is modelled in Figure 3. The effect of shale anisotropy is again strong in all cases and comparable to that obtained for dry fractures. These examples clearly show that shale anisotropy has to be taken into account for any form of AVO analysis. These effects are quite significant even for weak anisotropy. 
(A)

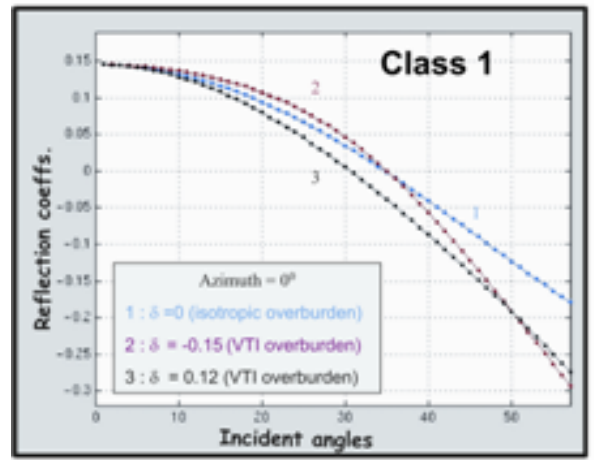

(C)

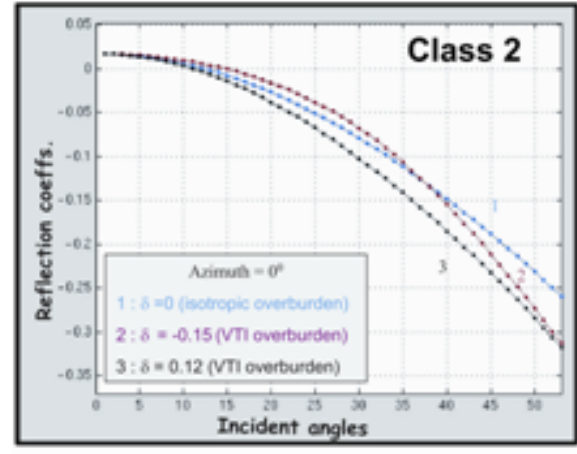

(E)

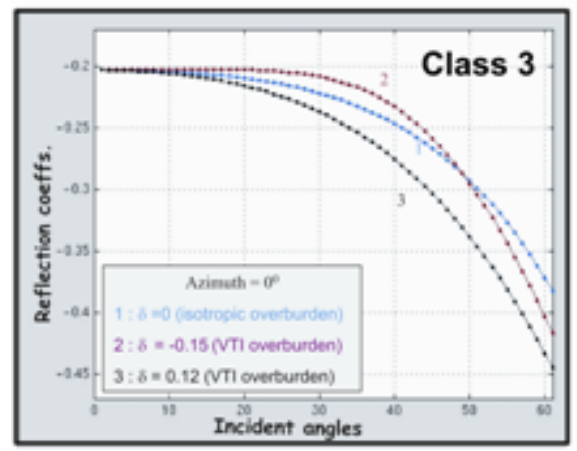

(B)

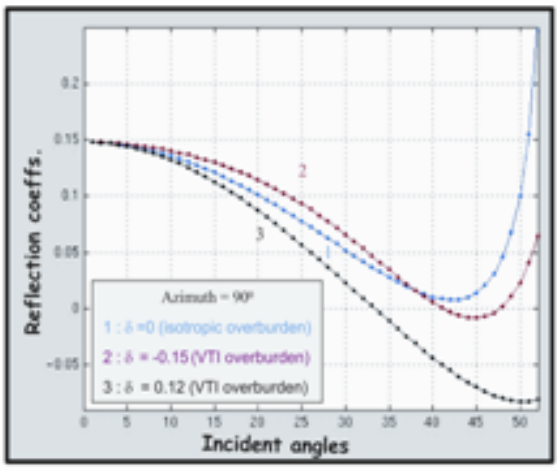

(D)

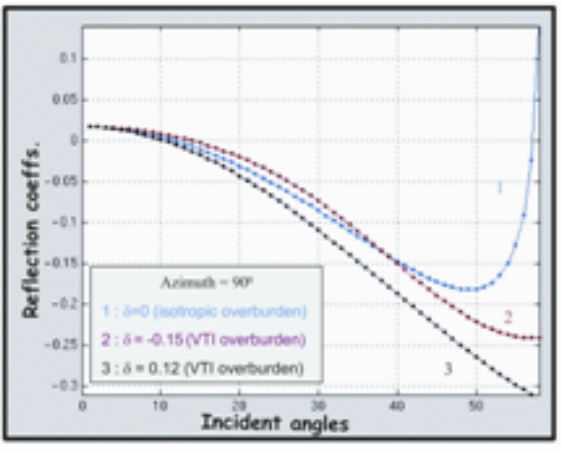

(F)

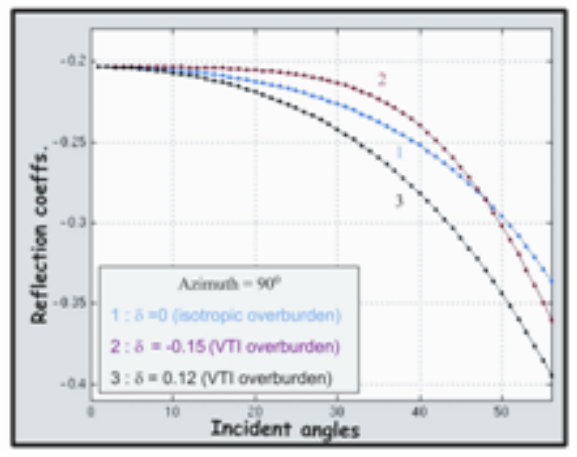

Figure 2 P-wave AVO response for shale/sand models (classes 1, 2 and 3). Isotropic shale/HTI sand is shown in blue, VTI shale $(\delta=-0.15)$ over HTI sand is shown in red and VTI shale $(\delta=0.12)$ over HTI sand is shown in black. Dry fractures are modeled $(\mathrm{ZN} / \mathrm{ZT}=0.95)$ : (a) class 1 , normal to fractures (b) class 1 , along to fractures, (c) and (d) class 2, normal and along fractures and (e) and (f) class 3 , normal and along fractures. 
(A)

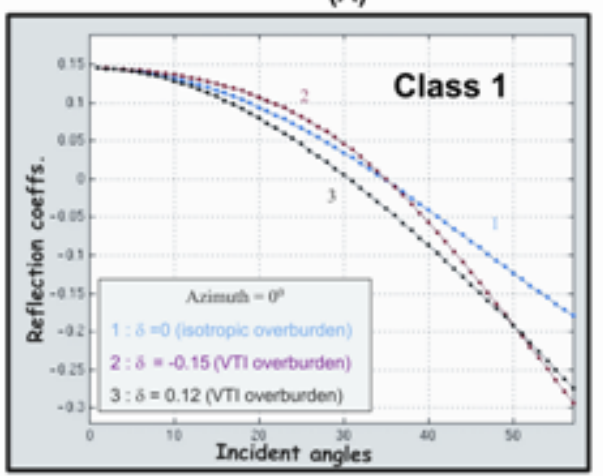

(C)

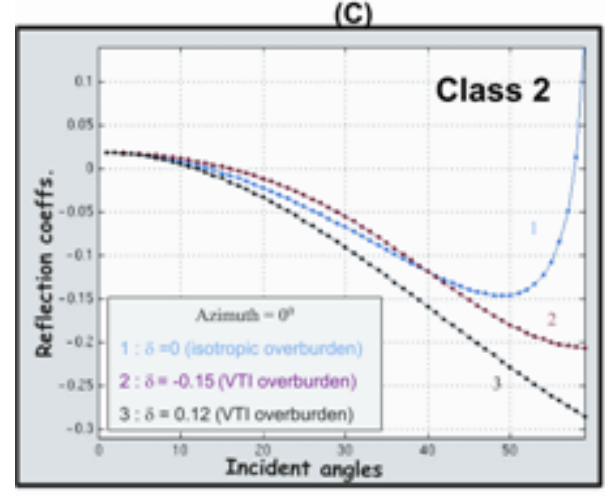

(E)

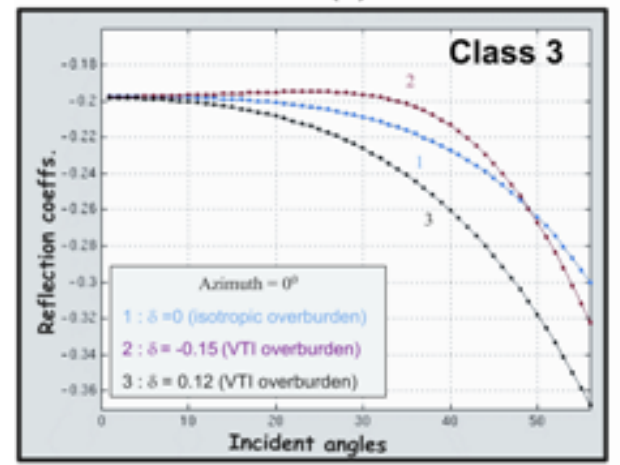

(B)

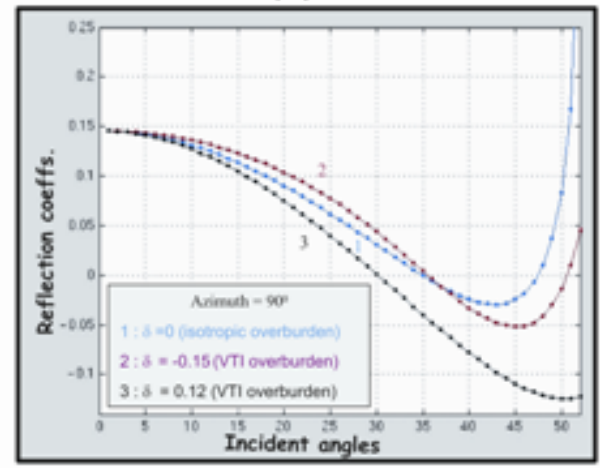

(D)

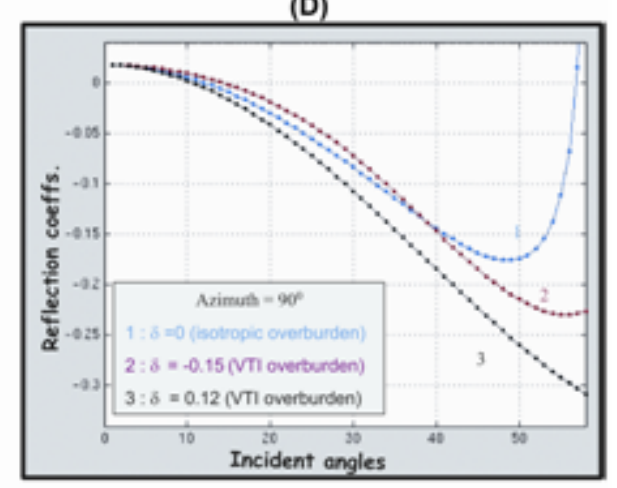

(F)

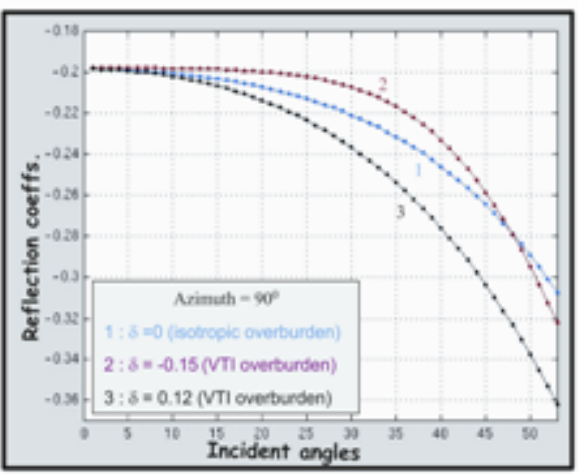

Figure 3 P-wave AVO response for shale/sand models (classes 1, 2, and 3). Isotropic shale/HTI sand is shown in blue, VTI shale $(\delta=-0.15)$ over HTI sand is shown in red and VTI shale $(\delta=0.12)$ over HTI sand is shown in black. Fluid saturated fractures are modeled $(\mathrm{ZN} / \mathrm{ZT}=0.05)$ : (a) class 1 , normal to fractures (b) class 1, along to fractures, (c) and (d) class 2, normal and along fractures and (e) and (f) class 3, normal and along fractures. 


\subsection{Influence of Fracture Porosity on Azimuthal AVO Response}

Fracture porosity is defined as fracture density times mean fracture volume. This parameter is directly related to the fracture density it is of interest to examine its direct effect on AVO response by keeping the aspect ratio fixed.

(A)

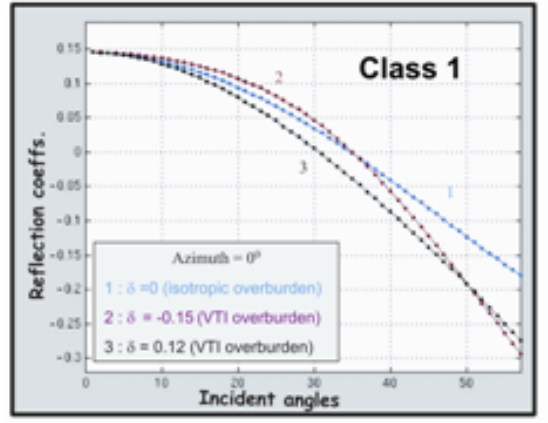

(C)

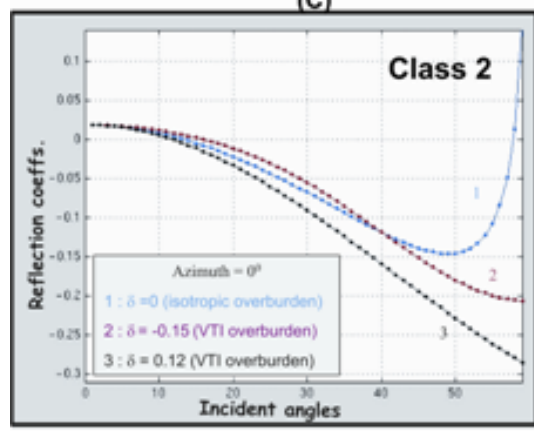

(E)

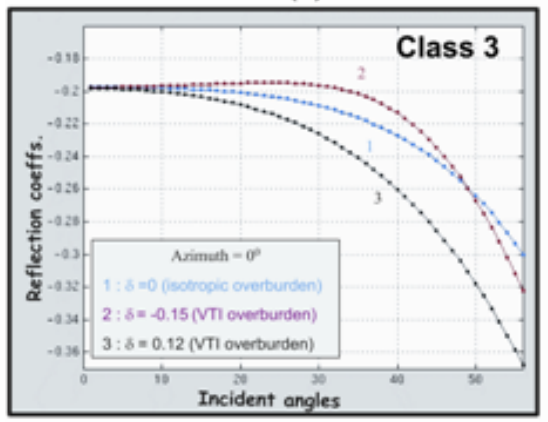

(B)

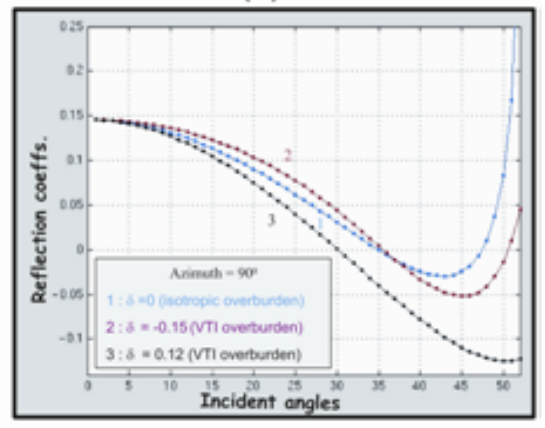

(D)

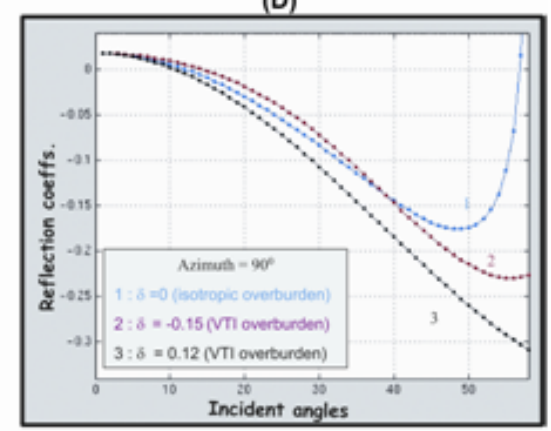

(F)

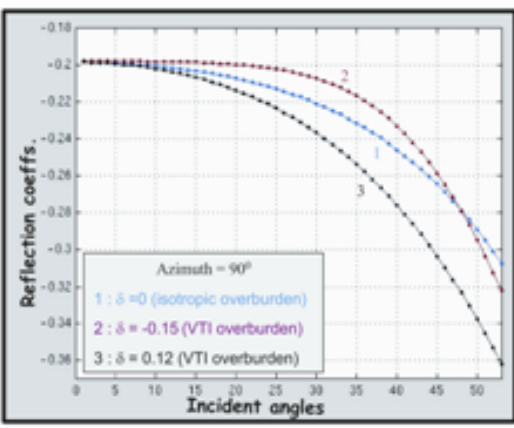

Figure 4 P-wave azimuthal AVO analysis over water-saturated vertically fractured sands for different fracture porosities (a) azimuth $=0^{0}$, (b) azimuth $=$ $45^{\circ}$, (c) azimuth $=90^{\circ}$ and (d) reflection coefficients versus fracture porosity computed for four incidence angles orthogonal to the fracture direction. 
(a)

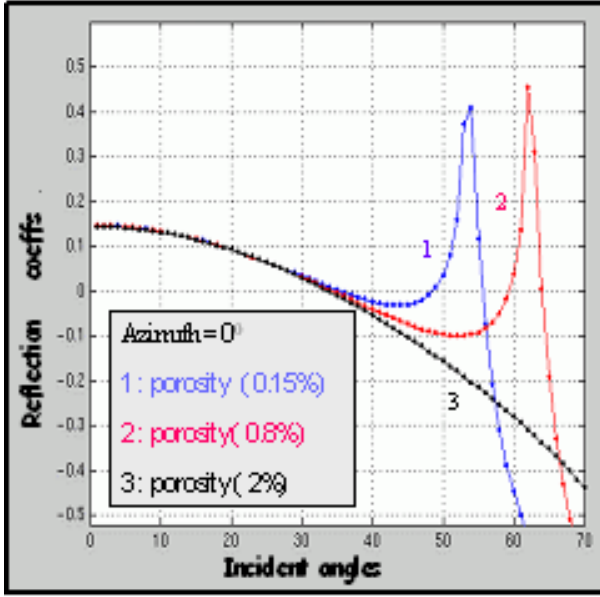

(c)

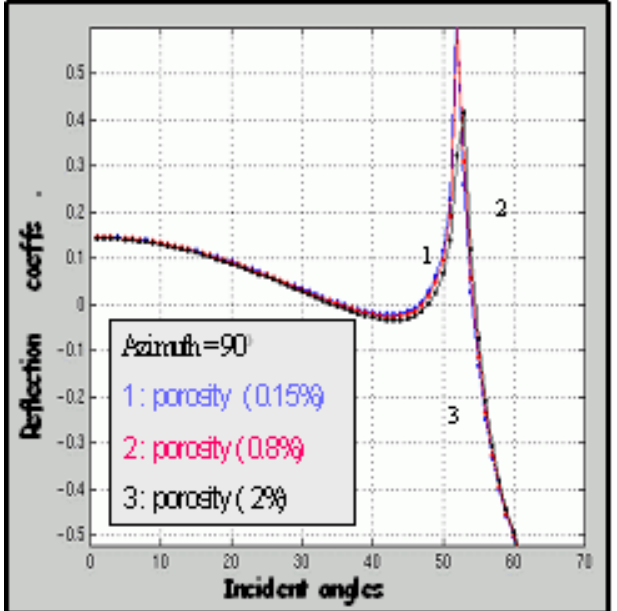

(b)

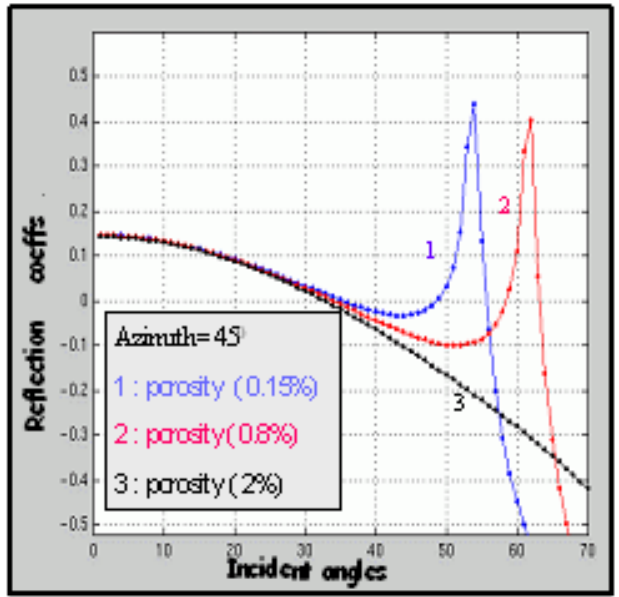

(d)

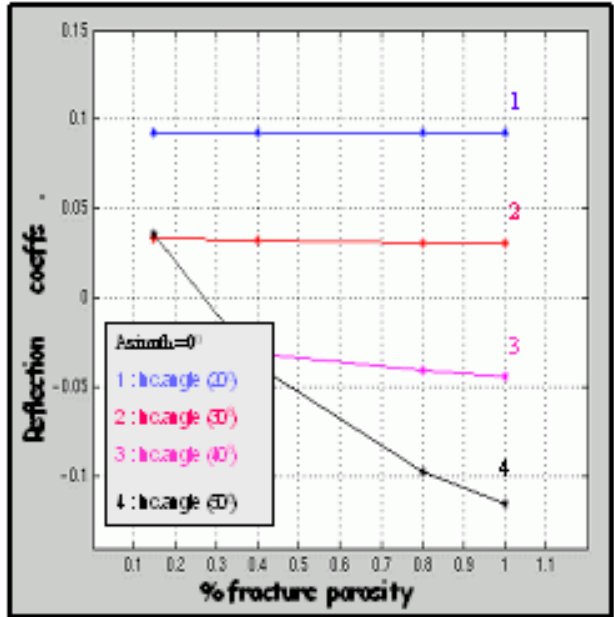

Figure 5 P-wave azimuthal AVO analysis over dry vertically fractured sands for different fracture porosities (a) azimuth $=0^{\circ}$, (b) azimuth $=45^{\circ}$, (c) azimuth $=$ $90^{\circ}$ and (d) reflection coefficients versus fracture porosity computed for four incidence angles orthogonal to fracture direction.

Such hypothetical case may arise if a reasonable assumption about aspect ratio could be made from other information such as geological or core sample analysis. To examine the characteristic information of fracture porosity on $\mathrm{P}$ wave azimuthal AVO responses, simple shale/fracture sand models were utilised. The elastic properties of the top layer were kept constant while elastic 
properties of the sand layer, representing a reservoir, varied with different fracture porosities. Calculations were carried out for both dry and fluidsaturated fractures having porosities of $0.15 \%, 0.8 \%$ and $2 \%$, respectively. The fracture aspect ratio was fixed at 0.05 .

The effect of fracture porosity on P-wave azimuthal AVO response for fluidfilled inclusions is shown in Figure 4. Overall the effect is small for moderate incidence angles. The difference is seen at large incidence angles as a shift in critical angle, shown by the peak. Similar effects are observed for dry fractures in the Figure 5.

\subsubsection{Influence Fracture Porosity on Thomsen's Anisotropic Parameters}

Figures 6.a-b show the effects of variable fracture porosity on Thomsen's anisotropic parameters for fluid filled and dry fractures with fracture porosities ranging from $0 \%$ up to $1 \%$. Assuming that the background of fractures was to be isotropic and an aspect ratio was fixed with 0.01 .

For fluid filled fractures P-wave anisotropy is mildly affected and assumes a maximum value of $10 \%$. However shear wave anisotropy is significantly affected. For dry fractures the difference in P-wave anisotropy is over $45 \%$ between low and high fracture porosities and overall much higher than shear wave anisotropy. These values of P-wave anisotropy seem very high compared with anisotropy measured in field and laboratory data.

(a)

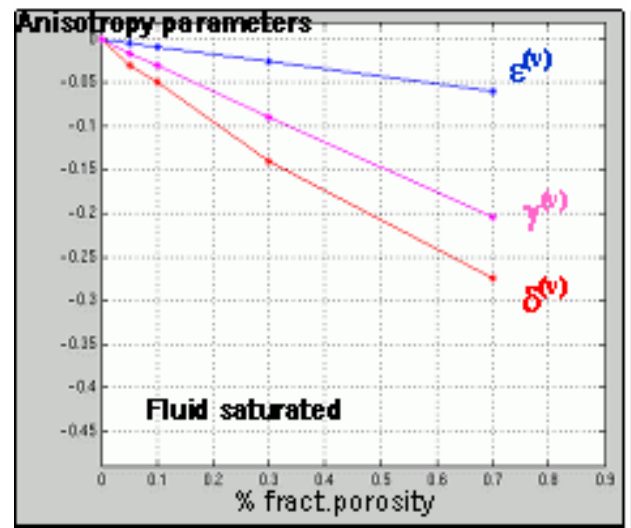

(b)

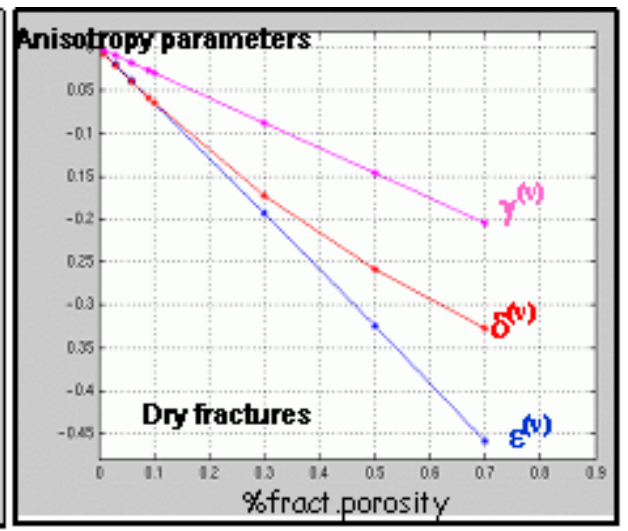

Figure 6 Variations of Thomsen's anisotropic parameters with fracture porosity for: (a) fluid saturated and (b) dry fractures. 


\section{Conclusions}

The effects of a VTI overburden can change reflection responses for small angles of incidence $\left(<20^{\circ}\right)$; although the overburden is weakly anisotropic.

The effect of variable fracture porosity on P-wave azimuthal AVO response shows small for moderate incidence angles. The difference is seen at large incidence angles as a shift in critical angle, shown by the peak.

For fluid filled fractures P-wave anisotropy is small affected; whilst for dry fractures the difference in P-wave anisotropy is high (over 45\%) between low and high fracture porosities. This suggests that assumption of overburden isotropy may cause severe distortions.

\section{Acknowledgment}

Part of this research is funded by ITB Research Grant no 0076/K01.03/PL2.1.5/VI/2005. The writer also acknowledges support from Geophysical Engineering Study Program FIKTM ITB.

\section{References Cited}

[1] Backus, M., Long-wave elastic anisotropy produced by horizontal layerin, J. Geophys. Res., 67, 4427-4440 (1962).

[2] Bakulin, A., Grechka, V. \& Tsvankin, I., Estimation of fracture parameters from reflection seismic data - Part 1: HTI model due to a single fracture set, Geophysics, 65, 1788-1802 (2000).

[3] Crampin, S., Effective anisotropic elastic constants of wave propagation through cracked solids, Geophys. J. Roy. Astr. Soc., 76, 133-145 (1984).

[4] Hudson, J.A., Wave speed and attenuation of elastic waves in material containing cracks, Geophys. J. Roy. Astr. Soc., 64, 133-150 (1981).

[5] Sayers, C.M. and Rickett, J.E., Azimuthal variation in AVO response for fractured gas sands, Geophys. Prosp., 45, 165-182 (1997).

[6] Schoenberg, M., Elastic wave behavior across linear slip interfaces, J. Acoust. Soc. Am., 68, 1516-1521 (1980).

[7] Schoenberg, M., and Muir, F., A calculus for finely layered anisotropic media, Geophysics, 54, 581-589 (1989).

[8] Schoenberg, M. and Protazio, J.S., Zoeppritz rationalized and generalized to anisotropy, Journal Seismic Exploration, I, 125-144 (1992).

[9] Schoenberg, M., and Sayers, C. M., Seismic anisotropy of fractured rock, Geophysics, 60, 204-211 (1995). 